\title{
Ohmic Heating for Tofu Making-A Pilot Study
}

\author{
Cheng-Chang Lien1, Yu-Chieh Shen1, Ching-Hua Ting2* \\ ${ }^{1}$ Department of Biomechatronic Engineering, National Chiayi University, Chiayi, Chinese Taipei \\ ${ }^{2}$ Department of Mechanical and Energy Engineering, National Chiayi University, Chiayi, Chinese Taipei \\ Email: *cting@mail.ncyu.edu.tw
}

Received January 2014

\begin{abstract}
The aim of this study is to explore the relationship between temperature and electrical conductivity of soya milk under ohmic heating in tofu making. The soya milk of 10 Brix was heated to a steady temperature of $90^{\circ} \mathrm{C}$. The applied voltage was increased and the temperature rising rate was investigated for adequate heating profiles in tofu making. Experimental results showed that the electrical conductivity of soya milk is proportional to the heating time. The temperature rising rate was increased from $1.46^{\circ} \mathrm{C}$ to $3.82^{\circ} \mathrm{C} / \mathrm{min}$ as a result of increased voltage. Hence ohmic heating could be an efficient, convenient heating measure in tofu making.
\end{abstract}

\section{Keywords}

\section{Soya Milk; Ohmic Heating; Electrical Conductivity; Temperature Rising Rate}

\section{Introduction}

Tofu is a salt- or acid-coagulated water-based gel, with soya lipids and proteins as well as other constituents trapped in gel networks [1]. It is made by coagulating soya milk-followed by either pressure or heat treatment after the addition of a coagulant. The taste of tofu is significantly affected by its final texture [2], i.e., by the firmness of the tofu gel's structure. The textural property is determined by the concentration of soya milk, the type and concentration of coagulant, the gelating pressure and temperature, and the gelation time [3].

The quality of a tofu can be examined using destructive testing. A textural analyzer is suitable for characterizing the mechanical property; while microscope analysis explores the chemical composition and the microstructure $[4,5]$. The above two methods are of a destructive nature and require laboratory practice. They are not suitable for on-line application as a quality indicator. Ultrasound which can give a big amount of information in a short time has been shown to be effective in detecting the gelation process and the final product in an efficient way [3,5]. While ultrasound is a means feasible for on-line quality detection, its setup is expensive.

The formation of a tofu gel requires soya milk mixed with a coagulant to be heated to a reaction temperature. In gelation, protein clusters are formed as a mechanism of ion exchanging between soya milk and the added coagulant. The concentration of the exchanging ions, anions orcations, is a result of the ongoing gelation process. The ion concentration can be easily characterized as electrical conductivity, an easy, economic, and efficient ${ }^{*}$ Corresponding author. 
measurement [6].

The objective of this study is to investigate the feasibility of using ohmic heating in tofu making with electrical conductivity as a quality indicator. Adequate temperature rising rates of soya milk is examined by applying different voltages onto the soya milk. The temperature profile can be used in tofu making with heat treatment and the electrical conductivity response can be for feedback control of gelation.

\section{Ohmic Heating for Tofu Making}

\subsection{Mechanism of Tofu Formation}

Addition of a coagulant into heated soya milk triggers the formation of tofu gels. The formation is a two-step mechanism [1]: soya proteins are denatured and then aggregated by the coagulant. Soya beans contain $35 \%$ $40 \%$ of storage and whey proteins by weight. The 7S protein, conglycinin, and $11 \mathrm{~S}$ protein, glycinin, are the two major proteins that affect the property of a tofu produce [7]. Figure 1 depicts the mechanism of soya milk transforming to tofu gels. When soya milk is heated to $65^{\circ} \mathrm{C}$ and beyond, the oil body is released. This is the first step of transformation. The $7 \mathrm{~S}$ and $11 \mathrm{~S}$ proteins will escape from the subunits at $75^{\circ} \mathrm{C}$ and $80^{\circ} \mathrm{C}$, respectively. Subunits are surrounded by protein particles with a size of 100nm [8-10]. The transformation completes.

\subsection{Principle of Ohmic Heating}

The performance of ohmic heating in food processing depends on the electrical conductivity of the foodstuff. DC (direct current) ohmic heating has power conduction by the moving ions in the foodstuff fluid. The viscosity of the fluid decreases in response to a hotter fluid. This speeds up the ionic motion and hence the electrical conductivity. The food fluid consists of conductive and resistive components which in turn, convert the electric current into heat. Figure 2 shows a model of soya milk under ohmic heating. The traditional heating method conveys heat from the heat generator to the heart of the medium through heat convection or conduction. A temperature gradient forms in traditional heating and leading therefore to a non-uniform temperature distribution, i.e. some medium may be overheated. Ohmic heating distributes electrical energy uniformly in the medium by means of the free ions and hence the medium is uniformly heated. The electrical conductivity of the processed medium follows [6]:

$$
\sigma=\frac{I L}{V A}
$$

with $\sigma$ in $\mathrm{Sm}^{-1}$ the electrical conductivity of the medium, $I$ in A the applied current, $V$ in $\mathrm{V}$ the applied voltage, $L$ in $m$ the distance between two electrodes, and $A$ in $m^{2}$ the contacting area between the medium and the electrodes.

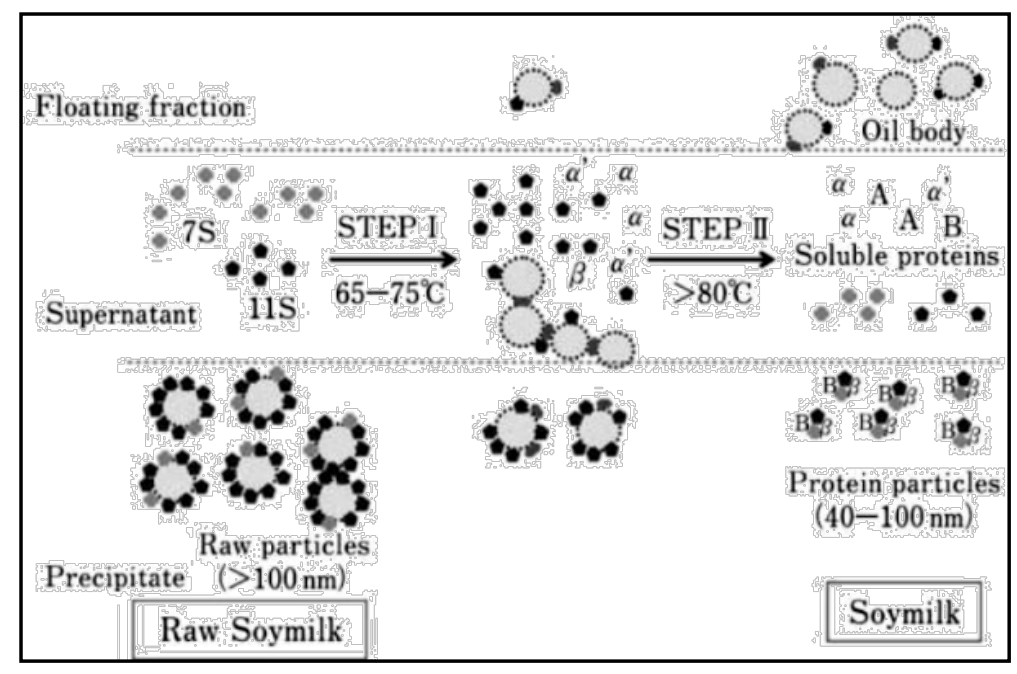

Figure 1. Process of protein separation in heated soya milk [9]. 


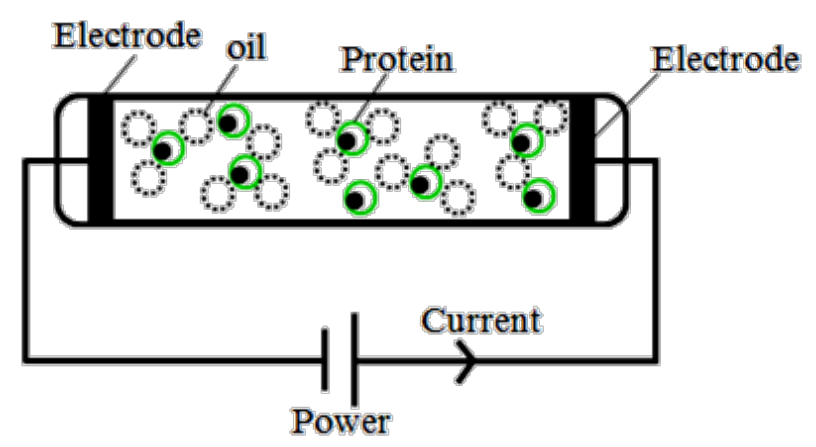

Figure 2. Soya milk under ohmic heating [11].

\subsection{Circuit model of Ohmic Heating}

Ohmic heating for fluid can be represented with an equivalent circuit model as shown in Figure 3. The resistor stands for the intrinsic electrical conductivity of the soya milk. It converts electrical energy from the voltage source $(V)$ to heat. The capacitor describes the capacitive effect built by the two electrode plates with the soya milk as the electrolyte in between. The circuit has the following first-order dynamics:

$$
I=\frac{V}{R}+C \frac{d v}{d t}
$$

\subsection{Factors Effecting Ohmic Heating}

An ohmic heating system heats a medium by applying an electrical field into the medium. A voltage $(V)$ applied on the two electrode plates forms the electric field $(E)$ with the correlation $E=V / l$ and the energy density stored in the medium between the two electrodes is $u=1 / 2 \epsilon E^{2}$ with $\epsilon$ the permititivity constant of the soya milk. The electrical power dissipated by the medium is:

$$
P=I^{2} \cdot R
$$

The steady state of Equation (2) indicates that a larger voltage application will result in a larger current. A larger current heats the medium in an efficient and powerful way. While the temperature of the soya milk increases, the electrical conductivity of the soya milk increases in a positive trend [6]:

$$
\sigma_{T}=\sigma_{\text {ref }} \cdot\left[1+m \cdot\left(T-T_{\text {ref }}\right)\right]
$$

where $\sigma_{T}$ is the electrical conductivity at current temperature $T$ and $\sigma_{r e f}$ is the electrical conductivity at the reference temperature $T_{\text {ref. }}$. Ions in an electrolyte are easily to escape from the substance molecules. Different substances will have different properties and hence the $\sigma_{T}$ is substance-specific [12-14].

\section{Materials and Methods}

\subsection{Materials}

Washed soya beans (800 g) were soaked for 8 - 12 hrs at a constant temperature $\left(30^{\circ} \mathrm{C}\right)$ in reverse osmosis (RO) water. One time by weight of $\mathrm{RO}$ water was added to the equivalent dry weight of the soya beans. The mixtures were ground and centrifugally filtered for 2 min to remove the residue. The soya milk produce was adjusted to a concentration of $10^{\circ}$ Brix. The procedure of preparing soya milk is summarized as Figure 4.

\subsection{Equipment}

Figure 5 illustrates the setup for ohmic heating experiments. Soya milk is placed in a steel container $(\mathrm{L} * \mathrm{~W}: 11$ $* 7 \mathrm{~cm}^{2}$ ) with ceramic coating. The two titanium electrodes are powered with a DC power supply (PSW-720, Instek, Taiwan) which is remotely controlled by a computer using the LabVIEW software (V8.3, National Instrument, USA). The soya milk is continuously stirred for uniform heat distribution. The temperature of the soya 


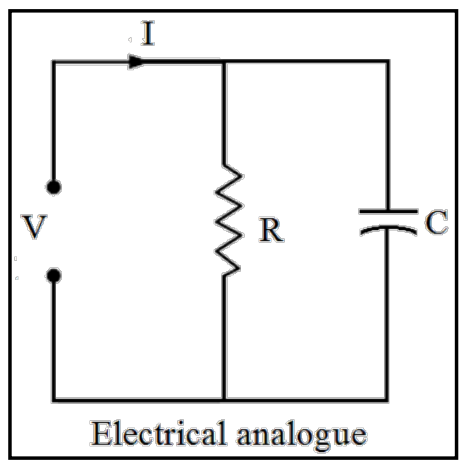

Figure 3. The equivalent circuit model of ohmic heating.

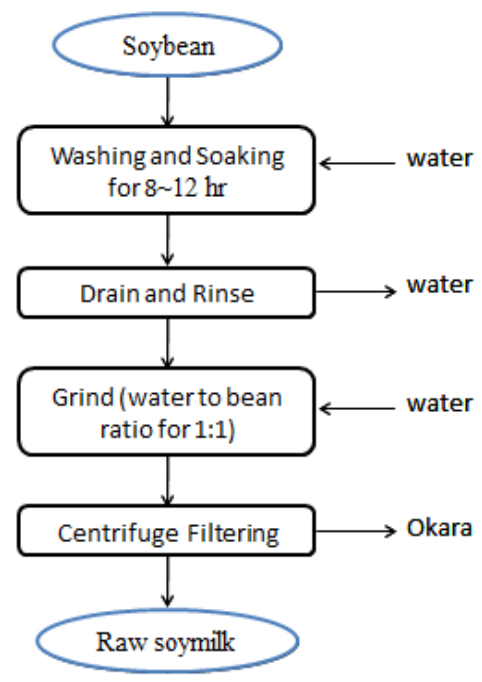

Figure 4. Procedure of soya milk preparation.

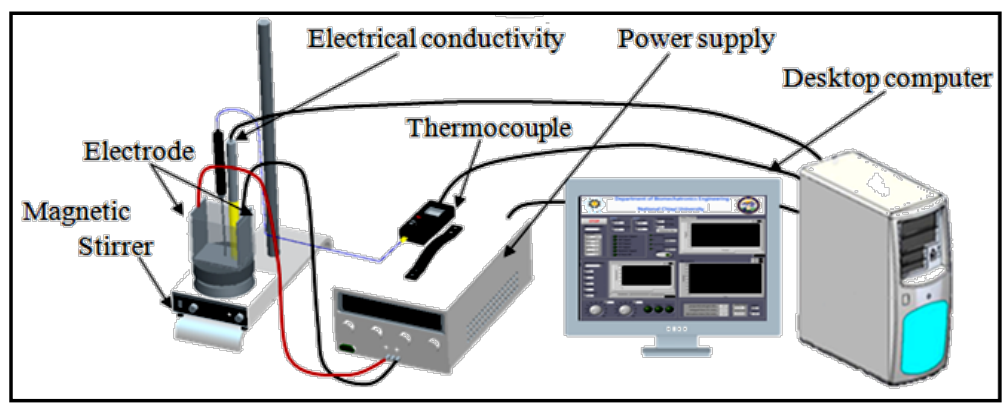

Figure 5. The ohmic heating setup.

milk is detected using a TM-925 temperature transducer (Prosperous, Taiwan) and the electrical conductivity is picked on line using by an electrical conductivity meter (Con 10, Eutch, Singapore).

\section{Results and Discussion}

\subsection{Effect of Voltage Application on Temperature Rising Rate}

The soya milk was heated to $90^{\circ} \mathrm{C}$ by ohmic heating with different voltage but the same current applications within 2 min. Figure 6 shows temperature responses to different applied voltages. A larger voltage results in a faster temperature rise. 


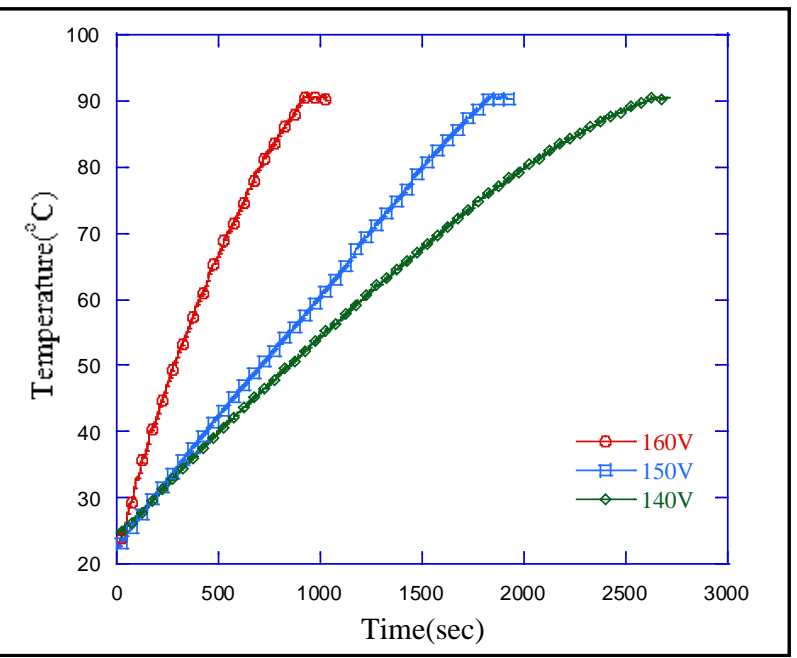

Figure 6. Temperature profiles of soya milks heated by different DC voltages.

Table 1 summarizes the temperature rising rates of different heating voltages. Clearly, increasing the voltage increases the temperature rising rate.

\subsection{Current Response of Ohmic Heating}

The temperature of the soya milk rises as a result of heating time in controlled current application. Figure 7 shows the current response at a temperature rising rate of $3.82^{\circ} \mathrm{C} / \mathrm{min}$. The current was large at the beginning as dominated by the first-order dynamics of Equation (2).

\subsection{Electrical Conductivity in Ohmic Heating}

Figure 8 shows measured and calculated electrical conductivities. There is a big discrepancy in between. This may be caused by the error of current measurement. By referring to Figure 7, there is large fluctuation in the current measurement.

\subsection{Electrical Conductivity of Different Applied Voltages}

Figure 9 shows calculated electrical conductivities of three voltage levels. The estimate is positively proportional to the applied voltage level. When the temperature is between 22 and $60^{\circ} \mathrm{C}$, the electrical conductivity of the soya milk has a positive temperature effect. Afterwards, it saturates.

This first-order dynamics effect is caused by the electro-osmotic effect [6]. Only in the beginning, a large amount of ions and salt in the soya solid dissolves in the milk.

\section{Conclusion}

In this paper, we have presented the potential application of combing ohmic heating and electrical conductivity measurement in tofu making by heat treatment. In theory, the soya milk can be uniformly cooked by ohmic

Table 1. Temperature rising rates of different applied voltages.

\begin{tabular}{cccc}
\hline$\Delta \mathrm{T}$ & \multicolumn{3}{c}{ Applied voltage(V) } \\
\cline { 2 - 4 }$\left({ }^{\circ} \mathrm{C} / \mathrm{min}\right)$ & 140 & 150 & 3 \\
\hline Samples & 3 & 3 & $3.82^{\mathrm{a}}$ \\
Mean & $1.46^{\mathrm{c}}$ & $2.16^{\mathrm{b}}$ & 0.61 \\
\hline Std & 0.24 & 0.7 & 0.7 \\
\hline
\end{tabular}

${ }^{1}$ Mean \pm Std. with different superscripts in the same row are significantly different $(\mathrm{P}<0.05)$, using the Scheffe test. 


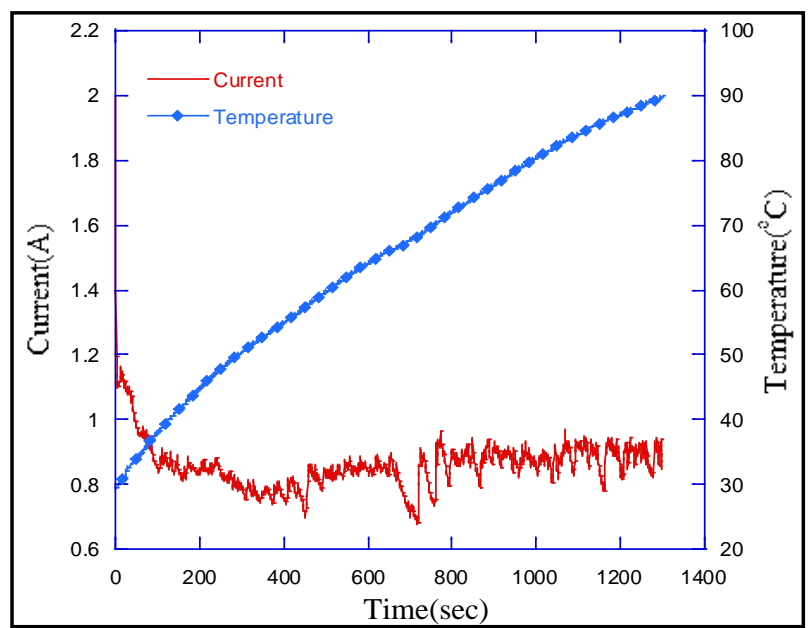

Figure 7. Heating current for a temperature rising rate of $3.82^{\circ} \mathrm{C} / \mathrm{min}$.

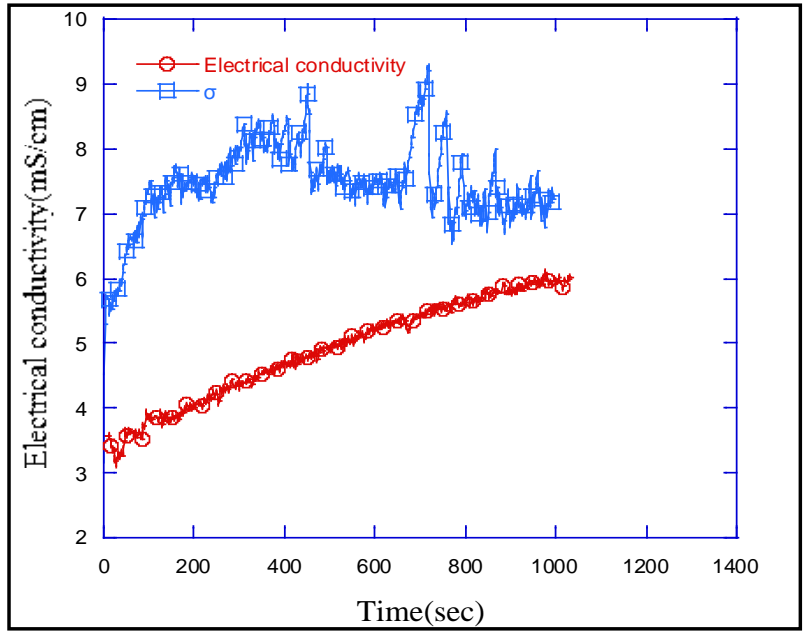

Figure 8. Measured and calculated electrical conductivities.

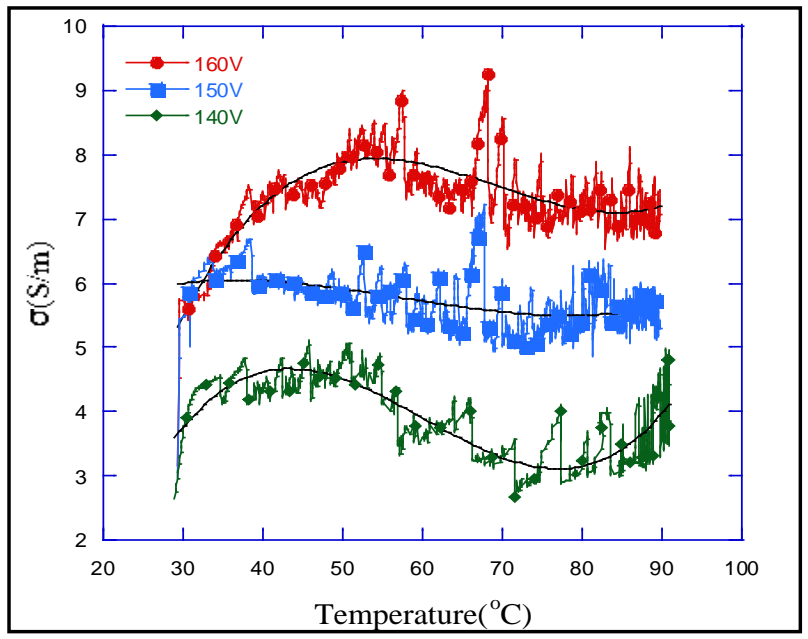

Figure 9. Calculated electrical conductivities at different voltage levels. 
heating. The electrical conductivity responses to different voltage levels show clear cuts around $60^{\circ} \mathrm{C}$, at this temperature the oil body of the soya milk is released. At temperatures around $75^{\circ} \mathrm{C}$ and $80^{\circ} \mathrm{C}$, the electrical conductivity response reaches a minimum saddle and starts to increase. These two temperatures correspond to the release of the 7S and 11S proteins, major proteins in tofu produce. Hence the electrical conductivity measurement could be a fast, convenient, and non-destructive testing method for on-line indication of tofu quality.

\section{Acknowledgements}

This work was financially supported by the National Science Council of Taiwan under the Grant No. 102-2221E-415-010.

\section{References}

[1] Kohyama, K., Sano, Y. and Doi, E. (1995) Rheological Characteristics and Gelation Mechanism of Tofu (Soybean Crud). Journal of Agricultural and Food Chemistry, 43, 1808-1812. http://dx.doi.org/10.1021/jf00055a011

[2] Jackson, C.-J., Dini, J.P., Lavandier, C., Rupasinghe, H.P.V., Faulkner, H., Poysa, V.,Buzzell, D. and DeGrandis, S. (2002) Effects of Processing on the Content and Composition of Isoflavones during Manufacturing of Soy Beverage and Tofu. Seminars in Food Analysis, 37, 1117-1123.

[3] Ting, C.H., Kuo, F.J., Lien, C.C. and Sheng, C.T. (2009) Use of Ultrasound for Characterising the Gelation Process in Heat Induced $\mathrm{CaSO}_{4}-2 \mathrm{H}_{2} \mathrm{O}$ Tofu Curd. Journal of Food Engineering, 93, 101-107. http://dx.doi.org/10.1016/j.jfoodeng.2009.01.015

[4] Saowapark, S., Apichartsrangkoon, A. and Bell, A.E. (2008) Viscoelastic Properties of High Pressure and Heat Induced Tofu Gels. Food Chemistry, 107, 984-989.

[5] Kuo, F.J., Lien, C.C., Huang, Y.Y. and Ting, C.H. (2011) Use of Ultrasound for Measuring Tofu Texture. Journal of Engineering in Agriculture, Environment and Food, 4, 83-89.

[6] Vicente, A.A. (2007) Ohmic Heating as an Alternative to Conventional Thermal Treatment. Dissertation for PhD Degree in Chemical and Biological Engineering, Universidade do Minho Escola de Engenharia.

[7] Wagner, J.R., Sobral, P.A. and Palazolo, G.G. (2010) Thermal Behavior of Soy Protein Fractions Depending on Their Preparation Methods, Individual Interactions, and Storage Conditions. Journal of Agriculture and Food Chemistry, 58, 10092-10100. http://dx.doi.org/10.1021/jf101957f

[8] Prestamo, G., Lesmes, M., Otero, L. and Arroyo, G. (2000) Soybean Vegetable Protein (Tofu) Preserved with High Pressure. Journal of Agricultural and Food Chemistry, 48, 2943-2947. http://dx.doi.org/10.1021/jf991251y

[9] Ono, T., Tezuka, M., Taira, H., Igarashi, Y. and Yagasaki, K. (2000) Properties of Tofus and Soy Milks Prepared from Soybeans Having Different Subunits of Glycinin. Journal of Agricultural and Food Chemistry, 48, 1111-1117. http://dx.doi.org/10.1021/jf9905601

[10] Guo, S.T. and Ono, T. (2005) The Role of Composition and Content of Protein Particles in Soymilk on Tofu Curding by Glucono- $\delta$-Lactone or Calcium Sulfate. Journal of Food Science, 70, 258-262. http://dx.doi.org/10.1111/j.1365-2621.2005.tb07170.x

[11] Li, X., Toyoda, K. and Ihara, I. (2011) Coagulation Process of Soymilk Characterized by Electrical Impedance Spectroscopy. Journal of Food Engineering, 105, 563-568. http://dx.doi.org/10.1016/j.jfoodeng.2011.03.023

[12] Amatore, C., Berthou, M. and Hébert, S. (1998) Fundamental Principles of Electrochemical Ohmic Heating of Solutions. Journal of Electroanalytical Chemistry, 457, 191-203. http://dx.doi.org/10.1016/S0022-0728(98)00306-4

[13] Pongviratchai, P. and Park, J.W. (2007) Electrical Conductivity and Physical Properties of Surimi-Potato Starch under Ohmic Heating. Journal of Food Science, 72, 1750-3841. http://dx.doi.org/10.1111/j.1750-3841.2007.00524.x

[14] Sastry, S.K., Sarang, S. and Knipe, L. (2008) Electrical Conductivity of Fruits and Meats during Ohmic Heating. Journal of Food Engineering, 87, 351-356. http://dx.doi.org/10.1016/j.jfoodeng.2007.12.012 